\title{
Urinary Tract Infections Identified in the Preoperative and Their Sensitivities to Antibiotics
}

\author{
Amadou Kassogué1 ${ }^{*}$, Fadima Tall², Daouda Diallo², Drissa Cissé1, Zanafon Ouattara ${ }^{3}$ \\ ${ }^{1}$ Department of Urology, University Hospital of Pr Bocar Sidy Sall, Kati, Mali \\ ${ }^{2}$ Department of Anaesthesia and Intensive Care, University Hospital of Pr Bocar Sidy Sall, Kati, Mali \\ ${ }^{3}$ Department of Urology, University Hospital of Gabriel Toure, Bamako, Mali \\ Email: *kassogueamadou@hotmail.fr
}

How to cite this paper: Kassogué, A., Tall, F., Diallo, D., Cissé, D. and Ouattara, Z. (2018) Urinary Tract Infections Identified in the Preoperative and Their Sensitivities to Antibiotics. Open Journal of Urology, 8 , 171-176.

https://doi.org/10.4236/oju.2018.85018

Received: February 15, 2018

Accepted: May 13, 2018

Published: May 16, 2018

Copyright $\odot 2018$ by authors and Scientific Research Publishing Inc. This work is licensed under the Creative Commons Attribution International License (CC BY 4.0).

http://creativecommons.org/licenses/by/4.0/

\begin{abstract}
Introduction: Urinary tract infection is a public health problem. Patients who have to undergo urology surgery are exposed to infectious complications if there is an untreated urinary tract infection. The objective of our study is to identify all the germs responsible for urinary tract infections discovered in the preoperative assessment of hospitalized patients with their susceptibility and antibiotic resistance profiles. Patients and Methods: This is a retrospective and descriptive study of 124 cases of cytobacteriological examination of urine collected over 2 years from January 1st, 2016 to December 31st, 2017, performed in the urology department of the University Hospital of Bocar S. Sall of Kati. The parameters studied were: age, sex, reason for admission, the diagnosis chosen, the result of the cytobacteriological examination of urine and the antibiogram. Results: We collected 124 cases of cytobacteriological examination of urine during this period. The average age of our patients was 58 years old with extremes ranging from 3 years to 84 years. The sex ratio was 6 in favor of men. 86 patients, or $69 \%$, were admitted for micturition disorders. In $53.2 \%$, the diagnosis was $\mathrm{BPH}$ followed by urolithiasis $22.6 \%$. Cytobacteriological examination of urine identified 55 cases of urinary infection, $44 \%$. In 34 cases or $60 \%$, the germ responsible was an E. coli. Two germs were sensitive only to one antibiotic. Conclusion: This study made it possible to have an idea about the antibiotic susceptibility of germs involved in urinary tract infections. Awareness-raising for the proper use of antibiotics needs to be strengthened to minimize the risk of increasing antibiotic resistance.
\end{abstract}

\section{Keywords}

Urinary Infection, ECBU, Antibiogram, Antibiotics 


\section{Introduction}

Urinary tract infection is a public health problem. The reference method for the microbiological diagnosis of urinary tract infection (UI) is the cytobacteriological examination of urine (ECBU), which directly measures bacteriuria and leukocyturia while allowing identification of the causative organism susceptibility. This antibiogram remains necessary after identification of the germ to study the sensitivity and resistance of the germ to antibiotics. UI is responsible for high morbidity and mortality worldwide. Patients who have to undergo surgery are exposed to infectious complications if there is an untreated UI. IU treatment is recommended to minimize this risk of complication, which can be fatal for the patient. The identification of the causative organisms and the determination of their resistance to antibiotics are necessary to rationalize the antibiotic treatment in order to avoid the development of resistance [1]. Struelens M. J. [2], reports that bacterial resistance to antibiotics is a natural adaptive phenomenon as old as the living world. The objective of our study is to identify all the germs responsible for urinary tract infections discovered in the preoperative assessment of hospitalized patients with their susceptibility and antibiotic resistance profiles.

\section{Patients and Methods}

This is a retro-prospective and descriptive study of 124 cases of ECBU collected over 2 years from January 1st, 2016 to December 31st, 2017, performed in the Urology Department of the Bocar S. Sall University Hospitalof Kati. It concerned all patients hospitalized during this period, whose urine samples were sent to the microbiology laboratory as part of the preoperative check-ups. The IU was defined by the presence of leukocyturia greater than or equal to $10^{4}$ elements $/ \mathrm{ml}$ and bacteriuria greater than $10^{5} \mathrm{CFU} / \mathrm{ml}$ to a single germ [1].

The parameters studied were: age, sex, reason for admission, the diagnosis retained, the result of the ECBU and the antibiogram.

\section{Results}

We collected 124 cases of urinary tract infection over a period of 2 years. The average age in our study was 58 years old with extremes ranging from 3 years to 84 years old. The sex ratio was 6 in favor of men. 86 patients, or $69 \%$, were admitted for voiding disorders (Table 1). In 53.2\%, the diagnosis was BPH followed by urolithiasis $22.6 \%$ (Table 2). ECBU identified 55 cases of urinary infection, i.e. $44 \%$. In 69 cases, $55.6 \%$, ECBU was negative. In 34 cases, or $60 \%$, the causative organism was an E. coli (Table 3). Two organisms were sensitive only to one antibiotic: Burkholderia cepacia, susceptible only to Cotrimoxazole and $E$. coli: sensitive only to Nitrofurantoin (Table 4). In 9 cases or $17 \%$ of the antibiogram performed, the germ was sensitive only to two antibiotics. Table 5 summarizes the observations on germs sensitive only to 2 antibiotics tested.

\section{Discussion}

Hailaji N.S.M. et al. [3], emphasizes that UI is a common pathology in the 
Table 1. Distribution of patients by reason of admission.

\begin{tabular}{ccc}
\hline Reason of admission & Effectifs & Percentage (\%) \\
\hline Micturition disorders & 86 & 69 \\
Lumbair pain & 27 & 22 \\
Hématuria & 5 & 4 \\
Scrotal pain & 6 & 5 \\
Total & 124 & 100 \\
\hline
\end{tabular}

86 patients, or $69 \%$, were admitted for micturition disorders.

Table 2. Distribution of patients according to the selected diagnosis.

\begin{tabular}{ccc}
\hline Diagnosis & Effectifs & Percentage (\%) \\
\hline BPH & 66 & 53.2 \\
Urinary stone: kidney, ureter, bladder & 28 & 22.6 \\
Prostate cancer & 7 & 5.6 \\
Ureteropelvic jonction & 4 & 3.2 \\
Urethra stenosis & 4 & 3.2 \\
Varicocele & 4 & 3.2 \\
Bladder cancer & 3 & 2.4 \\
Other & 8 & 6.5 \\
Total & 124 & 100.0
\end{tabular}

Other: bladder rupture, renal cyst, renal tumor, cervical sclerosis, scrotal abscess, cystocele, posterior urethral valve, torsion of the spermatic cord. A case of each of the pathologies mentioned.

Table 3. Distribution of patients according to the result of ECBU and according to the identified germs.

\begin{tabular}{ccc}
\hline Result of ECBU & Effectifs & Percentage (\%) \\
\hline Negative & 69 & 55.6 \\
E. coli & 34 & 27.4 \\
Candida albicans & 3 & 2.4 \\
Polymorphe & 3 & 2.4 \\
Klebsiella pneumoniae & 2 & 1.6 \\
Acinetobacter baumanii & 2 & 1.6 \\
Klebsiella oxytoca & 2 & 1.6 \\
Enterococcus & 1 & 0.8 \\
Burkholderia cepacia & 1 & 0.8 \\
Enterobacter cloacae & 1 & 0.8 \\
Pseudomonas aeruginosa & 1 & 0.8 \\
Staphylococcus aureus & 1 & 0.8 \\
Proteus mirabilis & 1 & 0.8 \\
Gram positive cocci & 1 & 0.8 \\
Gram negativebacillus & 1 & 0.6 \\
Total & 1 & 24 \\
\hline
\end{tabular}


Table 4. Distribution of germs according to their susceptibility to antibiotics.

\begin{tabular}{ccc}
\hline Antibiogramm & Effectifs & Percentage (\%) \\
\hline Sensitive germ with at least 3 antibiotics & 37 & 77 \\
Sensitive germ with 2 antibiotics & 9 & 17 \\
Sensitive germ only with a single antibiotic & 2 & 6 \\
Total & 48 & 100.0 \\
\hline
\end{tabular}

The two germs sensitive only to one antibiotic were: 1-Burkholderia cepacia: only susceptible to Cotrimoxazole; 2-E. coli: sensitive only to Nitrofurantoin.

Table 5. Summary of observations on germs sensitive only to 2 antibiotics tested.

\begin{tabular}{cccccc}
\hline & Age & Sex & Diagnosis & Germidentified & Antibiotic \\
\hline 01 & 73 & M & BPH & E. coli & $\begin{array}{c}\text { Imipenem, } \\
\text { Nitrofurantoin }\end{array}$ \\
02 & 83 & M & BPH & E. coli & Imipenm, Ertapenem \\
03 & 79 & M & BPH & E. coli & Imipenem, Cefoxitin \\
04 & 63 & M & BPH & E. coli & Imipenem, Amikacin \\
05 & 79 & M & BPH & E. coli & Cefixim, Gentamicin \\
06 & 42 & M & Kidney stone & E. coli & Ciprofloxacin, \\
07 & 64 & M & BPH & Proteus Mirabilis & Cefotaxin, Amikacin \\
08 & 51 & F & Kidney stone & Klebsiella & Gentamicin, Amikacin \\
09 & 76 & M & Urethrastenosis & BGN & Phenicols, Tetracyclines \\
\hline
\end{tabular}

community as well as in the hospital. An increase in antibiotic resistance of IU-causing organisms has been documented in several studies [3] [4] [5]. Inappropriate use of antibiotics is one of the causes of the emergence and increase of antibiotic resistance. The most frequently responsible germ is Escherichia coli. Hailaji N.S.M. et al. [3], found $64.4 \%$ in their study. Urinary tract infections are a real public health problem according to several studies [4] [5]. They are a frequent reason for consultation because they lead to an important and sometimes inappropriate prescription of antibiotics [5].

Lemaoui C. E [4], concluded that the bacteria have acquired in recent times, resistance to all classes of antibiotics currently used, sometimes posing major therapeutic problems. It is prudent to use these products with care to prolong their effectiveness [4]. Bacteria can easily develop a variety of antibiotic resistance mechanisms: either by selection of mutants or by acquisition of genetic material [2]. Of the 124 ECBUs completed, we identified 55 IU cases, 3 ECBUs were polymorphic. Among the 52 identified germs, 34 cases were E. coli or $65 \%$ of the identified germs. Several studies have shown that $E$. coli is the most common germ found in urinary tract infections [3] [5]. Our study is similar to that of Hailaji N.S.M. et al. [3], who found $64.4 \%$ E. coli in their study.

In urological surgery, when the surgical wound can be in contact with the urine, it is recommended to systematically perform a preoperative ECBU [6]. 
We carried out in this direction for hospitalized patients. Urinary tract infection is the most common infection, accounting for $25 \%$ of the elderly person's infections and the leading cause of nosocomial infection [6]. The average age in our study was 58 years old with extremes ranging from 3 years to 84 years old. Prostate pathology comes first with 66 cases among diagnoses accounting for $53 \%$ of our study. We are in the majority of the cases in front of elderly subjects often with other defects those which can complicate the clinical picture if it associates an untreated or abused UI. Conducting a microbiological urine analysis as part of a preoperative assessment remains common in daily practice. ECBU is the gold standard for positive diagnosis [6]. It is strongly recommended to screen for urinary colonization before an operation in contact with urine [7].

Bacterial resistance has a negative impact on the health of the population, increases the mortality and the side effects of the treatments but also the cost and the duration of the treatment. An infection of "toto-resistant" bacteria threatens the use of surgery and creates a vicious circle. Very few new antibiotics are in development [8]. Bissan, A.T. [1], conducted a study in infants aged 2 to 24 months, their study on antibiotic susceptibility of E. coli showed a high level of resistance to amoxicillin and amoxicillin-clavulanic acid and to trimethoprim sulfamethoxazole with $73.5 \%$ and $64.7 \%$ respectively [1].

We found that in our study two organisms were sensitive to only one antibiotic in our study: Burkholderia cepacia, sensitive only to Cotrimoxazole and $E$. coli, sensitive only to Nitrofurantoin. These two germs show resistance to antibiotics requiring special precautions. We did not find in our study any "resistant fungus" germs. We also found 9 germs sensitive only to two antibiotics or two families of antibiotics. Of these 9 germs, the 6 were an $E$. coli. We think that multi-resistant E. coli are beginning to appear in our situation.

The antimicrobial resistance phenomenon has accelerated and spread in the bacterial flora of animals and humans following the massive use of antibiotics in therapy and prophylaxis [2]. Antibiotic resistance increases the risk of treatment failure, adverse side effects, complication and extra care [2]. The threat of antibiotic resistance is global and new strategies are underway at the local, national and global levels. Fabre R et al. [9], studied the overall sensitivity of 1636 E. coli strains collected, they found a sensitivity to ciprofloxacin of $94 \%$ in women aged 15 to 65 years and $80 \%$ in humans. Resistance acquired is related to the level of antibiotic prescription in the populations studied [9]. A better knowledge of the bacterial ecology and the profiles of sensitivity of different bacteria is essential for better patient management. A preventive strategy, including hygiene and asepsis measures, the rationalization of the prescription of antibiotics to prevent the selection of multi-resistant germs is necessary.

\section{Conclusion}

This study allowed us to have an idea about the antibiotic resistance rates of germs involved in urinary tract infections. Awareness-raising action on the proper use of antibiotics needs to be strengthened to minimize the risk of anti- 
biotic resistance. Studies of this kind also make it possible to know the bacterial ecology in our service and the sensitivity to the antibiotics necessary to avoid cases of septicemia in per or postoperative immediate.

\section{Conflicts of Interest}

No conflict interest.

\section{Ethics Committee}

No ethics committee in our hospital.

\section{References}

[1] Bissan, A.T., Razine, R., Doumbia, M. and Benouda, A. (2016) Communityurinary Tract Infection: Bacteriology and Resistance in a Moroccan Infant Population. Revue d' Épidémiologie et de Santé Publique, 64, 139-140.

[2] Struelens, M.J., Denis, O., Rodriguez-Villalobos, H. and Hallin, M. (2007) The Threat of Antibiotic Resistance: Origin, Impact and Public Health Perspectives. Revue Medicale De Bruxelles, 28, 381-384.

[3] Hailaji, N.S.M., Ould Salem, M.L. and Ghaber, S.M. (2016) Sensitivity to Antibiotics Uropathogens Bacteria in Nouakchott-Mauritania. Progrès en Urologie, 26, 346-352. https://doi.org/10.1016/j.purol.2016.04.004

[4] Lemaoui, C.E., Layaida, H., Badi, A. and Foudi, N. (2017) Current Approaches to Fight against Antibiotic Resistance.Journal des Anti-Infectieux, 19, 12-19. https://doi.org/10.1016/j.antinf.2017.01.003

[5] Bentroki, A.A., Gouri, A., Yakhlef, A., Touaref, A., Gueroudj, A. and Bensouilah, T. (2012) Antibiotic Resistance of Strains Isolated from Community Acquired Urinary Tract Infections between 2007 and 2011 in Guelma (Algeria). Annales de Biologie Clinique, 70, 666-668.

[6] Molliex, S., Pierre, S., Bléry, C., Marret, E. and Beloeil, H. (2012) Routine Preinterventional Tests. Annales Françaises d Anesthésie et de Réanimation, 31, 752-763. https://doi.org/10.1016/j.annfar.2012.06.009

[7] Bruyere, F., Sotto, A., Escaravage, L., Cariou, G., Mignard, J.-P., Coloby, Hoznek, P.A., et al. (2010) Recommandations of the Infectious Diseases Committee of the French Association of Urology: Antibiotic Prophylaxis for Urological Procedures. Progrès Urologie, 20, 101-108.

[8] Laxminarayan, R., Duse, A., Wattal, C., Zaidi, A.K., Wertheim, H.F., Sumpradit, N., Vlieghe, E., Hara, G.L., et al. (2013) The Lancet. Infectious Diseases, 13, 1057-1098. https://doi.org/10.1016/S1473-3099(13)70318-9

[9] Fabre, R., Mérens, A., Lefebvre, F., Epifanoff, G., Cerutti, F., Pupin, H., et al. (2010) Susceptibility to Antibiotics of Escherichia coli Isolated from Community-Acquired Urinary Tract Infections. Médecine et Maladies Infectieuses, 40, 555-559. https://doi.org/10.1016/j.medmal.2010.03.002 